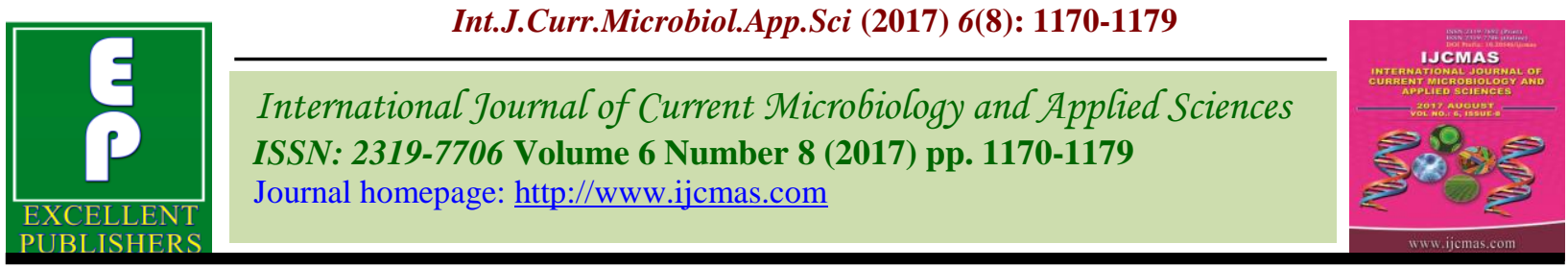

Original Research Article

https://doi.org/10.20546/ijcmas.2017.608.145

\title{
Studies on Mean Performance for Yield and its Contributing Traits of Sponge Gourd [Luffa cylindrica (Roem) L.]
}

\author{
Yamuna Prasad Singh*, V.B. Singh, Praveen Kumar Singh, \\ Vimlesh Kumar, Maneesh Pandey and Gyanendra Singh
}
Department of Vegetable Science, Narendra Deva University of Agriculture and Technology, Narendra Nagar, Kumarganj, Faizabad (U.P.) 224 229, India
*Corresponding author

\section{A B S T R A C T}

The experiment trial were conducted in two years with aims to determine average mean performance of sponge gourd involving 14 parental lines and their $40 \mathrm{~F}_{1}$ hybrids with three replications in RBD. Analysis of variances for line $\times$ tester mating design revealed that variance due to treatments, parents and crosses were highly significant for all the traits in both the years, variances due to parents $v s$. crosses were highly significant for all the characters except for vine length and fruit circumference in both the years and variances due to line vs. testers were highly significant for all the traits except for fruit circumference and average fruit weight in both the years along with some of the component traits in $\mathrm{Y}_{1}$ and $\mathrm{Y}_{2}$. Partitioning of variances into lines revealed highly significant differences for all the traits and variances due to testers were also highly significant for all the traits in both the years. The highest mean performance for fruit yield per plant $(\mathrm{kg})$ along with some of the component traits was exhibited by parents NDSG-21 (3.39 and 3.51), NDSG-12 (3.36 and 3.24), NDSG-1 (3.28 and 3.13), Pusa Chikni (3.24 and 3.18) and NDSG-15 (3.04 and 2.99 ) in both the years and some others genotypes given better performance in respects to fruit yield and other contributing traits. Highest yielding hybrids was NDSG-55 $\times$ NDSG$11(3.95$ and $4.23 \mathrm{~kg}$ ) followed by NDSG-63 $\times$ Pusa Chikni (3.95 and $4.35 \mathrm{~kg})$ and NDSG$24 \times$ Pusa Chikni (3.89 and $4.12 \mathrm{~kg}$ ) in $\mathrm{Y}_{1}$ and $\mathrm{Y}_{2}$,respectively, while some other parents and also hybrids given better performance in respects to fruit yield and other contributing traits. The above mentioned genotypes may be used as donor parents in hybridization programme for developing high yielding varieties of respective groups.

\section{Introduction}

Sponge gourd (Luffa cylindrica MJ. Roem (L.) syn. L. aegyptica Mill.) Is one of the most important cucurbit vegetable, which is grown rainy and summer season throughout the country and world? Its origin place is subtropical Asian region particularly India and it is domesticated species (Kaloo, 1993). It belongs to the family Cucurbitaceae with diploid chromosome number $2 \mathrm{n}=2 \mathrm{x}=26$. In
India, a number of major and minor cucurbits are cultivated which share about 5.6 per cent of the total vegetable production. The main goal of research on cucurbitaceous vegetables in India is to improve productivity on sustainable basis through developing biotic and abiotic resistant variety/hybrid coupled with quality attributes. The yield potential of cucurbits could be increased by adopting the 
standardized agro-techniques and plant protection measures. India being the second largest producer of vegetables in the world next only to China, shares about 15 per cent of the world output of vegetables from about 3 per cent of total cropped area in the country. The current production level is over 110 million tonnes from an area of 7.2 million hectares. The nutritive value of sponge gourd fruits per $100 \mathrm{~g}$ edible portion (tough skin removed, edible portion $80 \%$ ) is: water 93.2 $\mathrm{g}$, energy $18 \mathrm{kcal}$, protein $1.2 \mathrm{~g}$, fat $0.2 \mathrm{~g}$, carbohydrate $2.9 \mathrm{~g}$, fibre $2.0 \mathrm{~g}$, Ca $36 \mathrm{mg}, \mathrm{P}$ $19 \mathrm{mg}, \mathrm{Fe} 1.1 \mathrm{mg}$, carotene $120 \mu \mathrm{g}$, thiamine $0.02 \mathrm{mg}$, riboflavin $0.06 \mathrm{mg}$, niacin $0.4 \mathrm{mg}$ and the composition of young leaves per 100 $\mathrm{g}$ edible portion is: water $89 \mathrm{~g}$, protein $5.1 \mathrm{~g}$, carbohydrate $4.0 \mathrm{~g}$, fibre $1.5 \mathrm{~g}$, Ca $56 \mathrm{mg}$, Fe $11.5 \mathrm{mg}$, carotene $9.2 \mathrm{mg}$, ascorbic acid 95 mg (Holland et al., 1991). In spite of such a large production, the per capita per day supply of vegetables could not rise above 175 $\mathrm{g}$ in the country which is lower than the recommended dietary allowance (RDA) of 350 to $400 \mathrm{~g}$ per capita per day for a balanced diet (Rai and Pandey, 2007). The vegetable requirement of our country is estimated to be 220 million tonnes by 2020 (Singh, 2004). This target can best be achieved through use of improved varieties and hybrids technology in combination with superior crop management skills. It is a monoecious and highly cross pollinated crop in which a large amount of variations are observed for most of the economically important traits. Variability found in shape, size and colour of fruits is most conspicuous. The tender fruits are rich in vitamin $\mathrm{A}$, vitamin $\mathrm{C}$ and iron (Yawalkar, 2004) (Fig. 1).

In India little attention has been given for the genetic improvement of sponge gourd by collecting diverse germplasm, their morphological characterization and assessing the variability parameters. Although, the information on above aspects in sponge gourd is available, the literature pertaining to such aspects is relevant to the materials and environments used and can't be generalized. Therefore, further studies aimed at generating and comparing information on above aspects in sponge gourd are warranted to facilitate the development of high yielding hybrid cultivars as well as varieties.

\section{Materials and Methods}

In the present investigation 54 genotypes (10 lines, 4 testers and $40 \mathrm{~F}_{1}$ 's) were evaluated in Randomized Block Design with three replications at the Main Experiment Station of the Department of Vegetable Science, Narendra Dev University of Agriculture and Technology, Faizabad (U.P.) India, under two Zaid seasons during $2014\left(\mathrm{Y}_{1}\right)$ and $2015\left(\mathrm{Y}_{2}\right)$. The treatments were sown in rows spaced 2.50 meters apart with a plant to plant spacing of 0.50 meter. All the recommended agronomic package of practices, protection measures and recommended dose of manures and fertilizers were applied to raise a good crop. Observations were recorded on all the six plants maintained carefully in each plot for fourteen quantitative characters viz., node number to anthesis of first staminate flower, node number to anthesis of first pistillate flower, days to anthesis of first staminate flower, days to anthesis of first pistillate flower, node number of first fruit harvest, days to first fruit harvest, number of primary branches per plant, inter nodal length $(\mathrm{cm})$, vine length $(\mathrm{m})$, fruit length $(\mathrm{cm})$, fruit circumference $(\mathrm{cm})$, average fruit weight $(\mathrm{g})$, number of fruits per plant and average fruits yield per plant $(\mathrm{kg})$. Analysis of variance was carried out as suggested by Panse and Sukhatme (1967).

\section{Results and Discussion}

Analysis of variances for line $\times$ tester mating design revealed that variance due to 
treatments, parents and crosses were highly significant for all the traits in both the years, variances due to parents $v s$. crosses were highly significant for all the characters except for vine length and fruit circumference in both the years and variances due to line $v s$. testers were highly significant for all the traits except for fruit circumference and average fruit weight in both the years along with some of the component traits in $Y_{1}$ and $Y_{2}$.

Fig.1 Fruit shape of sponge gourd (parents and crosses)

Fig. I

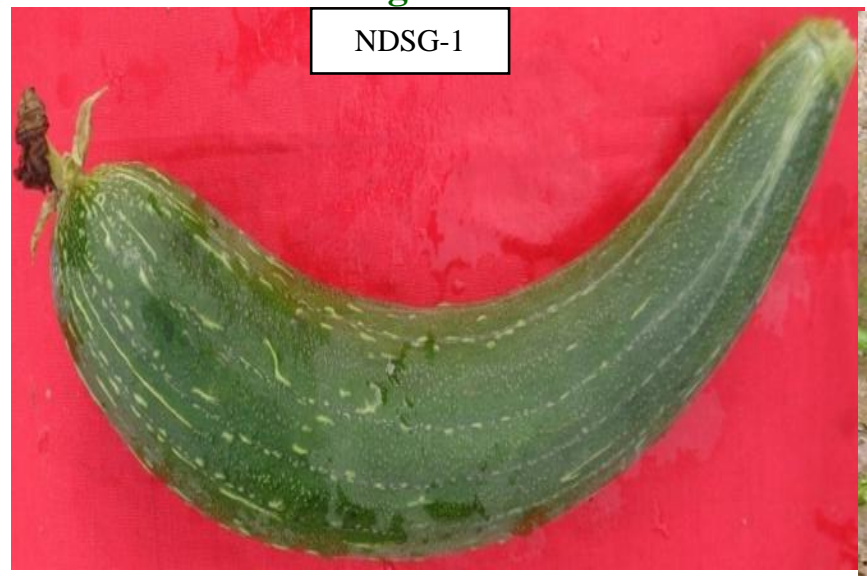

Fig. II

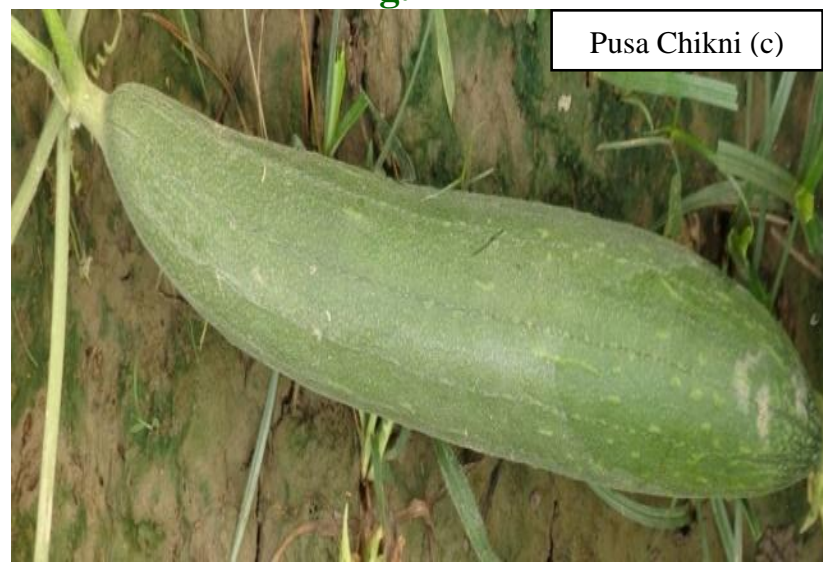

Fig. III

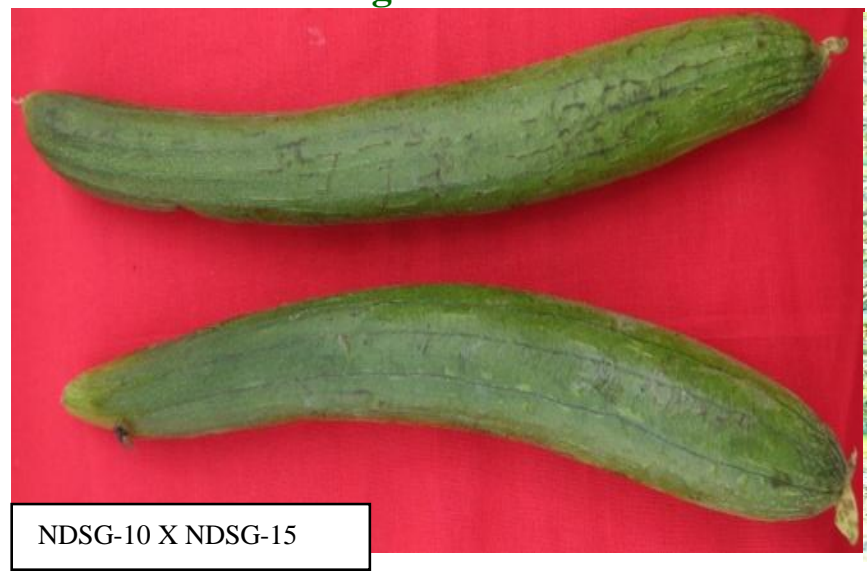

Fig. IV

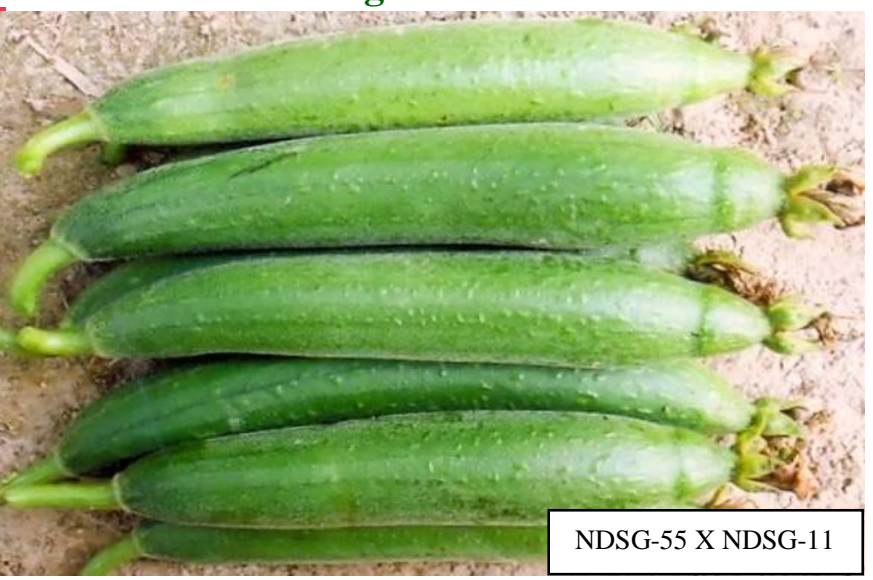

Fig. V

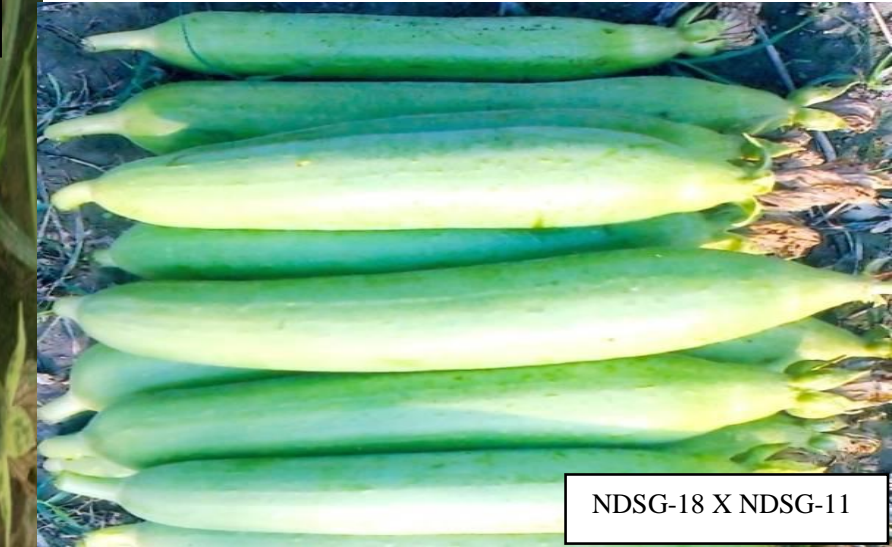

Fig. VI

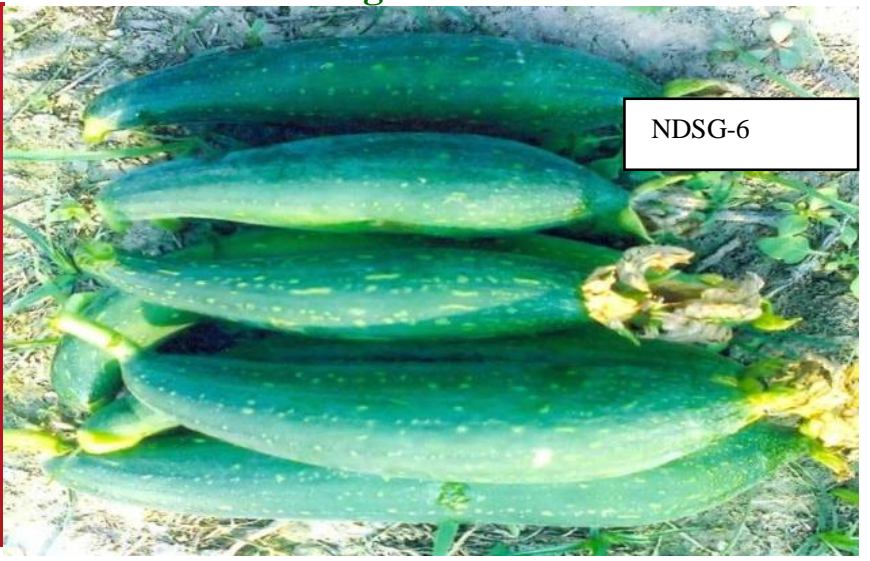


Table.1 Analysis of variance for 14 characters of line $\times$ tester set of crosses and their parents in sponge gourd $\left(\mathrm{Y}_{1}=2014\right.$ and $\left.\mathrm{Y}_{2}=2015\right)$

\begin{tabular}{|c|c|c|c|c|c|c|c|c|c|}
\hline Sources of variation & Years & $d f$ & $\begin{array}{l}\text { Node no.to } \\
\text { anthesis of first } \\
\text { staminate flower }\end{array}$ & $\begin{array}{l}\text { Node no.to } \\
\text { anthesis of } \\
\text { first pistillate } \\
\text { flower }\end{array}$ & $\begin{array}{l}\text { Days to } \\
\text { anthesis of } \\
\text { first } \\
\text { staminate } \\
\text { flower }\end{array}$ & $\begin{array}{l}\text { Days to } \\
\text { anthesis of } \\
\text { first pistillate } \\
\text { flower }\end{array}$ & $\begin{array}{l}\text { Node no. of } \\
\text { first fruit } \\
\text { harvest }\end{array}$ & $\begin{array}{l}\text { Days to first } \\
\text { fruit harvest }\end{array}$ & $\begin{array}{l}\text { No. of primary } \\
\text { branches per } \\
\text { plant }\end{array}$ \\
\hline \multirow{2}{*}{ Replications } & $\mathrm{Y}_{1}$ & 2 & 0.071 & 0.391 & 0.144 & 2.100 & 0.667 & 7.473 & 0.085 \\
\hline & $\mathrm{Y}_{2}$ & 2 & 0.098 & 0.563 & 3.376 & 1.381 & 0.347 & 8.749 & 0.159 \\
\hline \multirow{2}{*}{ Treatments } & $Y_{1}$ & 53 & $12.090^{* *}$ & $18.168^{* *}$ & $85.673^{* *}$ & $74.999 * *$ & $18.379 * *$ & $66.324 * *$ & $3.916^{* * *}$ \\
\hline & $\mathrm{Y}_{2}$ & 53 & $15.392 * *$ & $18.064 * *$ & $84.872 * *$ & $70.830 * *$ & $16.549 * *$ & $66.368 * *$ & $4.513 * *$ \\
\hline \multirow{2}{*}{ Parents } & $Y_{1}$ & 13 & $33.878^{* *}$ & $42.517 * *$ & $197.508^{* *}$ & $136.871^{* *}$ & $37.834 * *$ & $84.191 * *$ & $7.223^{* * *}$ \\
\hline & $\mathrm{Y}_{2}$ & 13 & $46.532 * *$ & $39.818^{* *}$ & $185.022 * *$ & $105.603^{* *}$ & $32.339 * *$ & $84.027 * *$ & $9.091 * *$ \\
\hline \multirow{2}{*}{ Parents (Line) } & $\mathrm{Y}_{1}$ & 9 & $38.097 * *$ & $30.224 * *$ & $171.692 * *$ & $145.092 * *$ & $23.855^{* *}$ & $92.169 * *$ & $8.538^{* * *}$ \\
\hline & $\mathrm{Y}_{2}$ & 9 & $50.992 * *$ & $20.829 * *$ & $166.769^{* *}$ & $91.122 * *$ & $19.438 * *$ & $70.653 * *$ & $9.681 * *$ \\
\hline \multirow{2}{*}{ Parents (Testers) } & $\mathrm{Y}_{1}$ & 3 & $20.758 * *$ & $93.538 * *$ & $339.645 * *$ & $157.816^{* *}$ & $91.236^{* *}$ & $65.762 * *$ & $3.645 * *$ \\
\hline & $\mathrm{Y}_{2}$ & 3 & $41.151^{* *}$ & $107.294 * *$ & $290.649 * *$ & $149.978^{* *}$ & $81.660 * *$ & $112.620 * *$ & $4.508^{* * *}$ \\
\hline \multirow{2}{*}{ Lines vs Testers } & $\mathrm{Y}_{1}$ & 1 & $35.264 * *$ & 0.094 & 3.438 & 0.052 & $3.438 * *$ & $67.680 * *$ & $6.120^{* * *}$ \\
\hline & $\mathrm{Y}_{2}$ & 1 & $22.541 * *$ & $8.288 * *$ & $32.425^{* *}$ & $102.811^{* *}$ & 0.486 & $118.614 * *$ & $17.527^{* *}$ \\
\hline \multirow{2}{*}{ Parents vs Crosses } & $Y_{1}$ & 1 & $149.504 * *$ & $198.483^{* *}$ & $539.444 * *$ & $327.576^{* *}$ & $213.811^{* *}$ & $251.287 * *$ & 0.044 \\
\hline & $\mathrm{Y}_{2}$ & 1 & $164.577 * *$ & $249.438 * *$ & $450.061 * *$ & $332.401 * *$ & $263.797 * *$ & $292.015 * *$ & $2.121 * *$ \\
\hline \multirow{2}{*}{ Crosses } & $\mathrm{Y}_{1}$ & 39 & $1.304 * *$ & $5.429 * *$ & $36.759 * *$ & $47.899 * *$ & $6.882 * *$ & $55.626^{* *}$ & $2.913^{* *}$ \\
\hline & $\mathrm{Y}_{2}$ & 39 & $1.187 * *$ & $4.879 * *$ & $42.124 * *$ & $52.532 * *$ & $4.946^{* *}$ & $54.695^{* *}$ & $3.049 * *$ \\
\hline \multirow{2}{*}{ Error } & $\mathrm{Y}_{1}$ & 106 & 0.131 & 0.321 & 3.728 & 4.021 & 0.319 & 7.322 & 0.152 \\
\hline & $\mathrm{I}_{2}$ & 106 & 0.121 & 0.312 & 3.52 & 4.161 & 0.317 & 7.084 & 0.184 \\
\hline
\end{tabular}

*, ** Significant at $5 \%$ and $1 \%$ probability levels, respectively.

Table.1 Cont....

\begin{tabular}{|c|c|c|c|c|c|c|c|c|c|}
\hline Sources of variation & Years & $d f$ & $\begin{array}{l}\text { Inter nodal } \\
\text { length }(\mathrm{cm})\end{array}$ & $\begin{array}{l}\text { Vine } \\
\text { length (m) }\end{array}$ & $\begin{array}{l}\text { Fruit length } \\
\text { (cm) }\end{array}$ & $\begin{array}{l}\text { Fruit } \\
\text { circumference }(\mathbf{c m})\end{array}$ & $\begin{array}{l}\text { Average fruit } \\
\text { weight (g) }\end{array}$ & $\begin{array}{l}\text { No. of fruits } \\
\text { per plant }\end{array}$ & $\begin{array}{l}\text { Average fruits } \\
\text { yield per plant (kg) }\end{array}$ \\
\hline \multirow{2}{*}{ Replications } & $\mathrm{Y}_{1}$ & 2 & 0.406 & 0.019 & 0.565 & 0.338 & 132.476 & 6.651 & 0.055 \\
\hline & $\mathrm{Y}_{2}$ & 2 & 0.001 & 0.068 & 0.082 & 0.364 & 23.152 & 2.112 & 0.017 \\
\hline \multirow{2}{*}{ Treatments } & $\mathrm{Y}_{1}$ & 53 & $5.785 * *$ & $3.777^{* * *}$ & $47.285^{* *}$ & $1.156^{* * *}$ & $1361.004 * *$ & $93.070 * *$ & $0.960^{* * *}$ \\
\hline & $\mathrm{Y}_{2}$ & 53 & $5.590 * *$ & $3.269 * *$ & $43.558 * *$ & $1.475^{* * *}$ & $1131.408 * *$ & $93.096^{* * *}$ & $1.163^{* * *}$ \\
\hline \multirow{2}{*}{ Parents } & $Y_{1}$ & 13 & $8.948 * *$ & $4.432 * *$ & $72.697 * *$ & $1.297 * *$ & $1761.850 * *$ & $106.041^{* *}$ & $1.041 * *$ \\
\hline & $\mathrm{Y}_{2}$ & 13 & $6.225 * *$ & $3.733^{* * *}$ & $62.647 * *$ & $1.865^{* * *}$ & $1612.218^{* *}$ & $100.914 * *$ & $1.019 * *$ \\
\hline \multirow{2}{*}{ Parents (Line) } & $\mathrm{Y}_{1}$ & 9 & $9.179 * *$ & $5.720 * *$ & $84.128 * *$ & $1.462 * *$ & $2249.789 * *$ & $145.799 * *$ & $1.114 * *$ \\
\hline & $\mathrm{Y}_{2}$ & 9 & $5.294 * *$ & $4.928^{* *}$ & 77.457 *** & $2.200^{* *}$ & $1865.727 * *$ & $138.422 * *$ & $1.179^{* * *}$ \\
\hline \multirow{2}{*}{ Parents (Testers) } & $\mathrm{Y}_{1}$ & 3 & $10.820 * *$ & $1.150^{* * *}$ & $53.310 * *$ & $1.216^{* * *}$ & $866.074 * *$ & $7.738 *$ & $0.504^{* * *}$ \\
\hline & $\mathrm{Y}_{2}$ & 3 & $10.236 * *$ & $0.538^{* * *}$ & $27.191 * *$ & $1.471 * *$ & $1316.520 * *$ & 2.987 & $0.382 * *$ \\
\hline \multirow{2}{*}{ Lines vs Testers } & $\mathrm{Y}_{1}$ & 1 & $1.259^{*}$ & $2.688^{* * *}$ & $27.977 * *$ & 0.061 & 57.720 & $43.136^{* * *}$ & $2.004 * *$ \\
\hline & $\mathrm{Y}_{2}$ & 1 & $2.577 * *$ & $2.561 * *$ & $35.729 * *$ & 0.032 & 217.728 & $57.128^{* *}$ & $1.481 * *$ \\
\hline \multirow{2}{*}{ Parents vs Crosses } & $\mathrm{Y}_{1}$ & 1 & $19.311 * *$ & 0.001 & 0.002 & 0.722 & $748.321 * *$ & $245.344 * *$ & $7.603^{* *}$ \\
\hline & $\mathrm{Y}_{2}$ & 1 & $28.473 * *$ & 0.132 & $38.217 * *$ & 0.012 & $1241.996 * *$ & $354.175^{* *}$ & $7.776^{* * *}$ \\
\hline \multirow{2}{*}{ Crosses } & $\mathrm{Y}_{1}$ & 39 & $4.384 * *$ & $3.656^{* *}$ & $40.027 * *$ & $1.120^{* * *}$ & $1243.099 * *$ & $84.841^{* * *}$ & $0.763^{* *}$ \\
\hline & $\mathrm{Y}_{2}$ & 39 & $4.791 * *$ & $3.195^{* * *}$ & $37.332 * *$ & $1.383 * *$ & $968.303 * *$ & $83.796^{* * *}$ & $1.042 * *$ \\
\hline \multirow{2}{*}{ Error } & $\mathrm{Y}_{1}$ & 106 & 0.197 & 0.063 & 2.238 & 0.296 & 60.431 & 2.599 & 0.046 \\
\hline & $\mathrm{Y}_{2}$ & 106 & 0.189 & 0.063 & 2.263 & 0.306 & 57.288 & 2.426 & 0.039 \\
\hline
\end{tabular}

$*, * *$ Significant at $5 \%$ and $1 \%$ probability levels, respectively. 
Table. 2 Mean performance, general mean, range, coefficient of variation, critical difference and standard error for 14 characters of line $\times$ tester set of $40 \mathrm{~F}_{1}$ 's and their 14 parents of sponge gourd $\left(\mathrm{Y}_{1}=2014\right.$ and $\left.\mathrm{Y}_{2}=2015\right)$

\begin{tabular}{|c|c|c|c|c|c|c|c|c|c|c|c|c|c|c|c|}
\hline \multirow[t]{2}{*}{ S. No } & \multirow[t]{2}{*}{ Genotypes } & \multicolumn{2}{|c|}{\begin{tabular}{|l|} 
Node no. to \\
anthesis of first \\
staminate flower
\end{tabular}} & \multicolumn{2}{|c|}{$\begin{array}{l}\text { Node no.to } \\
\text { anthesis of first } \\
\text { pistillate flower }\end{array}$} & \multicolumn{2}{|c|}{$\begin{array}{l}\text { Days to anthesis } \\
\text { of first } \\
\text { staminate } \\
\text { flower }\end{array}$} & \multicolumn{2}{|c|}{$\begin{array}{l}\text { Days to anthesis } \\
\text { of first pistillate } \\
\text { flower }\end{array}$} & \multicolumn{2}{|c|}{$\begin{array}{l}\text { Node no. of first } \\
\text { fruit harvest }\end{array}$} & \multicolumn{2}{|c|}{$\begin{array}{l}\text { Days to first } \\
\text { fruit harvest }\end{array}$} & \multicolumn{2}{|c|}{$\begin{array}{l}\text { No. of primary } \\
\text { branches per } \\
\text { plant }\end{array}$} \\
\hline & & $Y_{1}$ & $\mathbf{Y}_{2}$ & $\mathbf{Y}_{1}$ & $Y_{2}$ & $\mathbf{Y}_{1}$ & $Y_{2}$ & $\mathbf{Y}_{1}$ & $\mathbf{Y}_{2}$ & $Y_{1}$ & $\mathbf{Y}_{2}$ & $\mathbf{Y}_{1}$ & $\mathbf{Y}_{2}$ & $Y_{1}$ & $\mathbf{Y}_{2}$ \\
\hline $\mathbf{1}$ & NDSG-1 X NDSG-11 & 4.67 & 4.30 & 6.17 & 6.73 & 28.17 & 30.20 & 32.10 & 30.33 & 7.17 & 7.23 & 46.27 & 48.87 & 6.20 & 6.47 \\
\hline 2 & NDSG-1 X NDSG-12 & 3.90 & 4.10 & 7.13 & 7.03 & 32.13 & 33.17 & 38.20 & 38.23 & 7.60 & 7.27 & 50.60 & 48.53 & 4.20 & 4.27 \\
\hline 3 & NDSG-1 X NDSG-15 & 6.03 & 5.53 & 8.23 & 8.03 & 35.37 & 36.23 & 42.17 & 40.00 & 9.07 & 8.37 & 52.13 & 50.27 & 5.47 & 5.67 \\
\hline 4 & NDSG-1 X Pusa Chikni & 5.33 & 5.77 & 7.33 & 7.50 & 30.17 & 31.43 & 33.37 & 35.23 & 8.20 & 8.47 & 51.23 & 51.53 & 6.10 & 6.33 \\
\hline 5 & NDSG-2 X NDSG-11 & 5.80 & 5.47 & 8.43 & 8.57 & 34.33 & 32.30 & 34.53 & 31.87 & 8.50 & 8.80 & 46.10 & 44.80 & 6.87 & 7.00 \\
\hline 6 & NDSG-2 X NDSG-12 & 4.30 & 4.20 & 7.07 & 7.43 & 32.17 & 31.90 & 40.30 & 41.33 & 8.10 & 7.50 & 50.13 & 52.13 & 4.57 & 4.87 \\
\hline 7 & NDSG-2 X NDSG-15 & 5.47 & 5.27 & 8.50 & 8.27 & 35.40 & 36.67 & 37.07 & 36.30 & 10.03 & 10.27 & 47.50 & 46.40 & 5.13 & 5.23 \\
\hline 8 & NDSG-2 X Pusa Chikni & 4.63 & 4.53 & 11.07 & 10.13 & 32.30 & 34.13 & 38.53 & 37.27 & 11.97 & 10.63 & 50.17 & 51.27 & 6.60 & 6.50 \\
\hline 9 & NDSG-4 X NDSG-11 & 4.07 & 4.07 & 6.73 & 6.47 & 23.20 & 22.73 & 28.23 & 30.23 & 6.77 & 7.20 & 40.53 & 38.50 & 7.07 & 7.10 \\
\hline 10 & NDSG-4 X NDSG-12 & 4.13 & 4.30 & 5.97 & 5.07 & 29.20 & 30.20 & 32.23 & 30.17 & 6.13 & 8.27 & 45.67 & 47.37 & 5.03 & 5.17 \\
\hline 11 & NDSG-4 X NDSG-15 & 4.70 & 4.63 & 7.23 & 7.13 & 28.43 & 30.47 & 35.50 & 36.27 & 9.03 & 7.13 & 45.37 & 44.83 & 6.13 & 6.10 \\
\hline 12 & NDSG-4 X Pusa Chikni & 4.03 & 4.23 & 7.17 & 7.10 & 24.07 & 25.37 & 30.20 & 31.67 & 8.27 & 8.00 & 55.23 & 54.63 & 7.13 & 7.20 \\
\hline 13 & NDSG-6 X NDSG-11 & 4.57 & 5.23 & 7.17 & 7.50 & 28.60 & 27.47 & 30.27 & 28.53 & 7.27 & 7.53 & 41.27 & 39.30 & 8.17 & 8.10 \\
\hline 14 & NDSG-6 X NDSG-12 & 4.50 & 5.10 & 6.17 & 6.27 & 31.30 & 30.50 & 31.53 & 32.13 & 7.00 & 6.30 & 47.13 & 46.20 & 6.10 & 6.27 \\
\hline 15 & NDSG-6 X NDSG-15 & 5.13 & 5.40 & 7.07 & 6.07 & 32.33 & 33.47 & 36.00 & 37.57 & 7.03 & 7.40 & 42.40 & 41.43 & 6.47 & 6.40 \\
\hline 16 & NDSG-6 X Pusa Chikni & 4.57 & 4.47 & 7.33 & 7.43 & 27.27 & 26.07 & 33.00 & 32.20 & 8.47 & 8.20 & 43.43 & 44.50 & 7.33 & 7.20 \\
\hline 17 & NDSG-10 X NDSG-11 & 3.97 & 4.10 & 6.13 & 6.10 & 23.17 & 23.13 & 25.47 & 27.40 & 7.00 & 6.77 & 38.23 & 41.27 & 6.07 & 6.27 \\
\hline 18 & NDSG-10 X NDSG-12 & 3.03 & 3.07 & 6.10 & 6.27 & 28.40 & 27.77 & 34.23 & 36.17 & 7.03 & 6.87 & 40.10 & 42.33 & 5.10 & 4.83 \\
\hline 19 & NDSG-10 X NDSG-15 & 3.13 & 3.33 & 7.00 & 8.00 & 30.10 & 32.00 & 31.00 & 30.20 & 8.03 & 8.30 & 40.33 & 42.20 & 5.93 & 5.80 \\
\hline 20 & NDSG-10 X Pusa Chikni & 3.53 & 3.73 & 6.13 & 6.43 & 29.50 & 28.27 & 32.40 & 34.07 & 6.13 & 6.43 & 44.33 & 46.10 & 7.23 & 7.30 \\
\hline 21 & NDSG-18 X NDSG-11 & 3.87 & 3.53 & 6.10 & 6.37 & 21.17 & 21.67 & 25.80 & 23.87 & 6.13 & 7.13 & 38.17 & 40.63 & 7.50 & 7.87 \\
\hline 22 & NDSG-18 X NDSG-12 & 3.87 & 4.23 & 6.43 & 6.23 & 25.17 & 27.57 & 38.37 & 41.43 & 6.43 & 7.10 & 43.33 & 45.43 & 6.03 & 6.27 \\
\hline 23 & NDSG-18 X NDSG-15 & 4.13 & 4.03 & 8.03 & 8.23 & 25.73 & 27.27 & 31.50 & 32.30 & 8.70 & 8.37 & 43.33 & 45.47 & 7.13 & 7.53 \\
\hline 24 & NDSG-18 X Pusa Chikni & 4.00 & 4.33 & 7.23 & 7.20 & 26.37 & 27.23 & 35.23 & 34.37 & 7.30 & 7.70 & 45.57 & 47.47 & 8.43 & 8.60 \\
\hline 25 & NDSG-21 X NDSG-11 & 4.20 & 3.90 & 7.67 & 7.40 & 28.43 & 25.43 & 30.57 & 28.23 & 8.13 & 8.23 & 40.00 & 42.07 & 6.07 & 6.50 \\
\hline 26 & NDSG-21 X NDSG-12 & 4.50 & 4.67 & 12.20 & 11.63 & 30.07 & 32.23 & 38.17 & 36.43 & 13.30 & 12.40 & 50.33 & 50.33 & 4.87 & 4.60 \\
\hline 27 & NDSG-21 X NDSG-15 & 5.13 & 5.33 & 8.03 & 8.27 & 30.30 & 31.13 & 33.20 & 31.40 & 9.13 & 8.50 & 44.83 & 43.17 & 5.33 & 5.50 \\
\hline 28 & NDSG-21 X Pusa Chikni & 4.43 & 4.37 & 8.27 & 8.07 & 34.43 & 35.03 & 35.40 & 35.50 & 8.57 & 8.40 & 49.97 & 51.23 & 6.17 & 6.30 \\
\hline 29 & NDSG-24 X NDSG-11 & 4.10 & 3.80 & 7.03 & 7.17 & 26.37 & 24.50 & 26.23 & 25.50 & 9.13 & 7.23 & 41.23 & 40.33 & 7.10 & 7.07 \\
\hline 30 & NDSG-24 X NDSG-12 & 4.70 & 4.40 & 8.10 & 9.13 & 34.43 & 34.43 & 37.67 & 38.47 & 10.07 & 10.47 & 49.93 & 50.37 & 6.17 & 6.27 \\
\hline
\end{tabular}




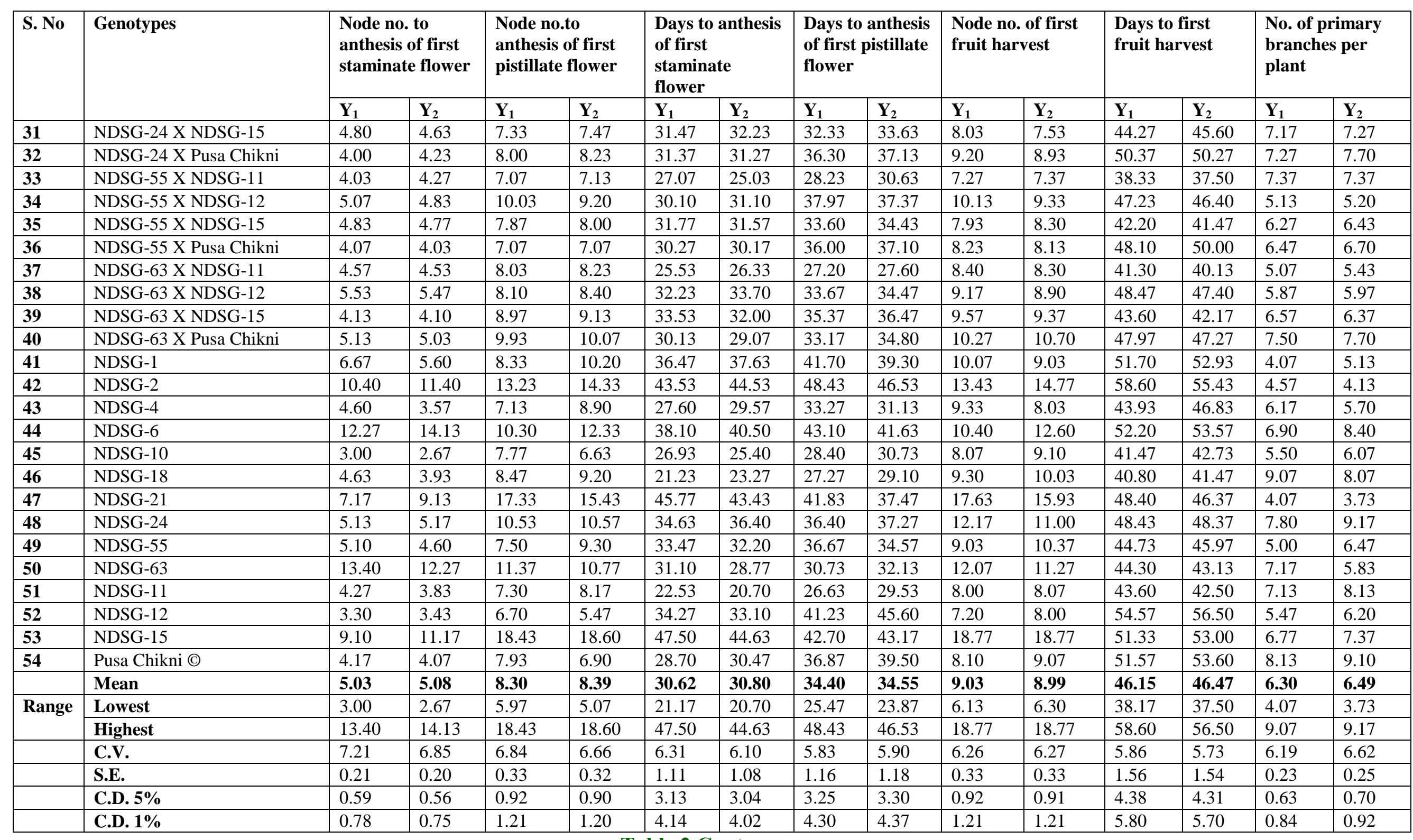

Table. 2 Cont......... 


\begin{tabular}{|c|c|c|c|c|c|c|c|c|c|c|c|c|c|c|c|}
\hline \multirow[t]{2}{*}{ S. No } & \multirow[t]{2}{*}{ Genotypes } & \multicolumn{2}{|c|}{$\begin{array}{l}\text { Inter nodal length } \\
(\mathrm{cm})\end{array}$} & \multicolumn{2}{|c|}{ Vine length (m) } & \multicolumn{2}{|c|}{ Fruit length $(\mathbf{c m})$} & \multicolumn{2}{|c|}{$\begin{array}{l}\text { Fruit } \\
\text { circumference }(\mathrm{cm})\end{array}$} & \multicolumn{2}{|c|}{$\begin{array}{l}\text { Average fruit } \\
\text { weight (g) }\end{array}$} & \multicolumn{2}{|c|}{$\begin{array}{l}\text { No. of fruits per } \\
\text { plant }\end{array}$} & \multicolumn{2}{|c|}{$\begin{array}{l}\text { Average fruits yield } \\
\text { per plant (kg) }\end{array}$} \\
\hline & & $\mathbf{Y}_{1}$ & $\mathbf{Y}_{2}$ & $\mathbf{Y}_{1}$ & $\mathbf{Y}_{2}$ & $\mathbf{Y}_{1}$ & $\mathbf{Y}_{2}$ & $\mathbf{Y}_{1}$ & $Y_{2}$ & $Y_{1}$ & $\mathbf{Y}_{2}$ & $\mathbf{Y}_{1}$ & $\mathbf{Y}_{2}$ & $\mathbf{Y}_{1}$ & $\mathbf{Y}_{2}$ \\
\hline 1 & NDSG-1 X NDSG-11 & 6.27 & 6.47 & 2.53 & 2.30 & 27.57 & 28.23 & 8.17 & 8.10 & 145.40 & 153.30 & 30.63 & 26.30 & 3.70 & 3.59 \\
\hline 2 & NDSG-1 X NDSG-12 & 6.00 & 6.20 & 3.03 & 3.10 & 25.23 & 26.23 & 9.57 & 9.63 & 161.57 & 160.23 & 23.23 & 21.27 & 3.26 & 2.91 \\
\hline 3 & NDSG-1 X NDSG-15 & 6.60 & 6.83 & 2.20 & 2.40 & 21.20 & 22.33 & 8.37 & 8.27 & 150.10 & 162.43 & 28.43 & 26.20 & 3.54 & 3.79 \\
\hline 4 & NDSG-1 X Pusa Chikni & 4.63 & 4.60 & 2.93 & 3.10 & 23.17 & 22.30 & 8.17 & 8.33 & 155.37 & 144.90 & 27.20 & 28.27 & 3.69 & 3.67 \\
\hline 5 & NDSG-2 X NDSG-11 & 6.73 & 6.50 & 2.60 & 2.63 & 31.23 & 32.17 & 8.07 & 8.13 & 131.23 & 135.47 & 25.63 & 24.43 & 3.09 & 2.96 \\
\hline 6 & NDSG-2 X NDSG-12 & 5.77 & 5.90 & 3.20 & 3.03 & 28.83 & 29.40 & 9.63 & 9.33 & 135.40 & 138.33 & 27.53 & 26.10 & 3.27 & 3.20 \\
\hline 7 & NDSG-2 X NDSG-15 & 8.37 & 8.67 & 2.43 & 2.43 & 24.17 & 25.03 & 8.33 & 8.37 & 140.37 & 148.30 & 22.27 & 22.13 & 2.61 & 2.81 \\
\hline 8 & NDSG-2 X Pusa Chikni & 4.13 & 4.37 & 3.93 & 3.33 & 22.20 & 23.37 & 8.37 & 8.23 & 145.10 & 145.10 & 17.27 & 16.47 & 2.17 & 2.08 \\
\hline 9 & NDSG-4 X NDSG-11 & 5.87 & 5.93 & 2.37 & 2.43 & 30.30 & 30.50 & 8.53 & 8.33 & 148.20 & 150.83 & 18.47 & 20.27 & 2.46 & 2.51 \\
\hline 10 & NDSG-4 X NDSG-12 & 5.83 & 5.77 & 5.67 & 5.50 & 26.93 & 28.53 & 9.43 & 9.37 & 157.83 & 145.33 & 21.53 & 22.03 & 2.86 & 2.69 \\
\hline 11 & NDSG-4 X NDSG-15 & 8.63 & 8.70 & 2.17 & 2.17 & 25.37 & 26.27 & 8.60 & 8.47 & 165.60 & 155.40 & 20.33 & 22.23 & 2.90 & 3.23 \\
\hline 12 & NDSG-4 X Pusa Chikni & 4.87 & 4.93 & 4.43 & 4.40 & 28.73 & 27.20 & 7.90 & 7.60 & 186.40 & 170.27 & 19.17 & 17.47 & 3.08 & 2.79 \\
\hline 13 & NDSG-6 X NDSG-11 & 6.90 & 6.77 & 2.90 & 2.93 & 32.50 & 33.00 & 9.13 & 9.17 & 150.40 & 145.20 & 25.37 & 26.43 & 3.31 & 3.39 \\
\hline 14 & NDSG-6 X NDSG-12 & 6.17 & 6.43 & 3.43 & 3.50 & 25.07 & 26.40 & 9.53 & 9.67 & 140.17 & 138.33 & 27.27 & 27.47 & 3.28 & 3.17 \\
\hline 15 & NDSG-6 X NDSG-15 & 8.70 & 8.80 & 3.07 & 3.40 & 27.37 & 23.70 & 9.83 & 9.67 & 150.50 & 150.57 & 24.40 & 25.30 & 3.23 & 3.36 \\
\hline 16 & NDSG-6 X Pusa Chikni & 6.30 & 6.63 & 3.30 & 3.17 & 25.23 & 26.30 & 9.23 & 9.17 & 174.70 & 190.10 & 20.27 & 18.20 & 3.04 & 2.92 \\
\hline 17 & NDSG-10 X NDSG-11 & 8.97 & 9.17 & 2.27 & 2.40 & 23.60 & 23.20 & 8.17 & 8.33 & 154.43 & 151.57 & 17.40 & 15.27 & 2.25 & 2.12 \\
\hline 18 & NDSG-10 X NDSG-12 & 7.50 & 7.87 & 3.60 & 3.47 & 19.27 & 20.27 & 9.73 & 9.93 & 180.57 & 165.60 & 17.27 & 20.27 & 3.03 & 2.87 \\
\hline 19 & NDSG-10 X NDSG-15 & 8.73 & 8.93 & 2.23 & 2.30 & 17.67 & 18.23 & 8.50 & 8.43 & 200.83 & 187.47 & 16.17 & 18.17 & 2.83 & 2.87 \\
\hline 20 & NDSG-10 X Pusa Chikni & 7.60 & 7.87 & 3.47 & 3.33 & 19.47 & 19.13 & 8.40 & 8.37 & 160.30 & 155.27 & 17.27 & 19.20 & 2.44 & 2.66 \\
\hline 21 & NDSG-18 X NDSG-11 & 6.40 & 4.87 & 2.50 & 2.57 & 23.33 & 24.10 & 9.30 & 9.63 & 125.07 & 120.63 & 37.63 & 38.60 & 3.87 & 4.01 \\
\hline 22 & NDSG-18 X NDSG-12 & 7.57 & 7.63 & 3.00 & 3.30 & 22.13 & 21.20 & 9.80 & 10.07 & 99.70 & 105.40 & 33.37 & 31.37 & 2.89 & 2.79 \\
\hline 23 & NDSG-18 X NDSG-15 & 8.23 & 8.37 & 2.50 & 2.70 & 20.13 & 21.43 & 9.07 & 9.07 & 115.53 & 125.17 & 32.77 & 31.03 & 3.06 & 3.45 \\
\hline 24 & NDSG-18 X Pusa Chikni & 7.10 & 7.30 & 5.30 & 4.97 & 23.30 & 24.20 & 8.57 & 8.20 & 118.33 & 130.57 & 34.43 & 37.50 & 3.79 & 4.06 \\
\hline 25 & NDSG-21 X NDSG-11 & 7.23 & 7.57 & 2.40 & 2.40 & 22.50 & 23.53 & 8.27 & 8.13 & 148.20 & 155.30 & 27.73 & 25.40 & 3.66 & 3.46 \\
\hline 26 & NDSG-21 X NDSG-12 & 7.60 & 7.47 & 3.63 & 3.47 & 21.03 & 23.17 & 9.27 & 9.37 & 135.73 & 141.97 & 27.27 & 24.97 & 3.16 & 3.12 \\
\hline 27 & NDSG-21 X NDSG-15 & 8.50 & 8.53 & 2.57 & 2.43 & 21.40 & 21.73 & 8.07 & 8.10 & 150.53 & 161.30 & 25.37 & 23.13 & 3.28 & 3.34 \\
\hline 28 & NDSG-21 X Pusa Chikni & 7.23 & 7.47 & 3.90 & 4.00 & 24.53 & 25.03 & 7.93 & 7.50 & 155.07 & 151.37 & 27.13 & 29.17 & 3.79 & 3.89 \\
\hline 29 & NDSG-24 X NDSG-11 & 7.23 & 7.20 & 3.07 & 3.23 & 22.13 & 23.30 & 8.63 & 8.63 & 115.43 & 130.53 & 26.10 & 23.33 & 2.67 & 2.48 \\
\hline 30 & NDSG-24 X NDSG-12 & 7.80 & 7.67 & 4.57 & 4.40 & 19.17 & 20.50 & 9.17 & 9.27 & 150.37 & 150.23 & 23.57 & 24.33 & 3.06 & 2.90 \\
\hline 31 & NDSG-24 X NDSG-15 & 8.30 & 8.43 & 5.33 & 5.03 & 18.43 & 19.43 & 8.37 & 8.27 & 130.27 & 121.57 & 22.23 & 24.20 & 2.56 & 2.45 \\
\hline 32 & NDSG-24 X Pusa Chikni & 7.50 & 7.60 & 6.13 & 5.93 & 19.17 & 20.43 & 8.53 & 8.33 & 163.23 & 170.50 & 26.30 & 25.33 & 3.89 & 4.12 \\
\hline 33 & NDSG-55 X NDSG-11 & 7.03 & 7.07 & 2.43 & 2.53 & 22.67 & 23.27 & 8.10 & 8.27 & 165.57 & 152.87 & 33.67 & 31.30 & 3.95 & 4.23 \\
\hline 34 & NDSG-55 X NDSG-12 & 7.67 & 7.40 & 3.53 & 3.67 & 20.20 & 20.33 & 9.67 & 9.83 & 138.23 & 131.20 & 29.70 & 32.27 & 3.67 & 3.53 \\
\hline 35 & NDSG-55 X NDSG-15 & 8.40 & 8.70 & 2.37 & 2.43 & 20.43 & 21.43 & 8.03 & 8.00 & 162.83 & 150.23 & 26.33 & 28.23 & 3.76 & 3.59 \\
\hline 36 & NDSG-55 X Pusa Chikni & 7.50 & 7.47 & 3.00 & 3.00 & 20.57 & 21.73 & 8.47 & 8.43 & 125.37 & 138.07 & 33.40 & 32.23 & 3.68 & 3.83 \\
\hline 37 & NDSG-63 X NDSG-11 & 6.13 & 6.33 & 6.37 & 6.43 & 27.47 & 28.07 & 9.27 & 9.37 & 123.50 & 110.40 & 24.73 & 26.33 & 2.39 & 2.49 \\
\hline 38 & NDSG-63 X NDSG-12 & 5.87 & 5.97 & 3.30 & 3.33 & 26.37 & 27.07 & 9.60 & 9.47 & 138.10 & 135.63 & 24.33 & 23.23 & 2.92 & 2.65 \\
\hline 39 & NDSG-63 X NDSG-15 & 8.77 & 8.63 & 3.00 & 3.20 & 25.37 & 24.37 & 9.20 & 8.77 & 132.03 & 130.23 & 24.23 & 23.23 & 2.76 & 2.47 \\
\hline 40 & NDSG-63 X Pusa Chikni & 5.83 & 5.93 & 3.03 & 3.20 & 24.50 & 23.53 & 9.03 & 9.10 & 153.33 & 160.23 & 28.17 & 30.20 & 3.95 & 4.35 \\
\hline 41 & NDSG-1 & 4.50 & 4.73 & 2.10 & 1.93 & 26.40 & 28.13 & 7.87 & 8.17 & 138.43 & 131.27 & 26.53 & 30.33 & 3.28 & 3.13 \\
\hline 42 & NDSG-2 & 3.57 & 4.10 & 4.33 & 4.03 & 30.07 & 31.00 & 8.10 & 7.87 & 126.20 & 118.30 & 21.23 & 19.33 & 2.36 & 2.52 \\
\hline 43 & NDSG-4 & 4.63 & 4.97 & 3.13 & 2.97 & 28.90 & 19.57 & 8.60 & 7.83 & 163.57 & 153.60 & 11.77 & 10.33 & 1.70 & 1.48 \\
\hline 44 & NDSG-6 & 6.43 & 6.23 & 3.00 & 3.30 & 31.23 & 29.30 & 9.83 & 8.33 & 153.50 & 160.23 & 22.47 & 21.33 & 2.84 & 2.95 \\
\hline
\end{tabular}




\begin{tabular}{|c|c|c|c|c|c|c|c|c|c|c|c|c|c|c|c|}
\hline 45 & NDSG-10 & 9.00 & 8.53 & 2.07 & 2.27 & 15.87 & 17.37 & 8.30 & 8.63 & 190.23 & 174.53 & 9.97 & 10.37 & 1.45 & 1.61 \\
\hline 46 & NDSG-18 & 7.33 & 6.60 & 5.33 & 5.10 & 22.07 & 24.27 & 9.20 & 8.80 & 95.53 & 100.60 & 31.23 & 29.20 & 2.60 & 2.67 \\
\hline 47 & NDSG-21 & 7.77 & 6.30 & 2.40 & 2.23 & 22.50 & 20.47 & 8.23 & 9.50 & 155.53 & 170.20 & 25.07 & 23.20 & 3.39 & 3.51 \\
\hline 48 & NDSG-24 & 7.73 & 7.17 & 6.17 & 5.93 & 18.80 & 20.10 & 8.40 & 9.27 & 150.60 & 139.37 & 18.37 & 20.13 & 2.47 & 2.61 \\
\hline 49 & NDSG-55 & 7.30 & 6.50 & 3.67 & 3.43 & 19.23 & 18.63 & 8.03 & 8.30 & 110.33 & 115.30 & 29.57 & 26.07 & 2.77 & 2.67 \\
\hline 50 & NDSG-63 & 5.57 & 4.97 & 2.90 & 3.10 & 27.50 & 28.33 & 9.67 & 10.60 & 129.83 & 125.87 & 23.20 & 20.80 & 2.53 & 2.36 \\
\hline 51 & NDSG-11 & 5.57 & 6.20 & 2.10 & 2.37 & 23.77 & 22.23 & 8.33 & 7.80 & 119.70 & 115.07 & 23.53 & 24.63 & 2.44 & 2.46 \\
\hline 52 & NDSG-12 & 5.60 & 5.77 & 3.50 & 3.33 & 20.77 & 20.73 & 9.57 & 9.40 & 144.80 & 150.47 & 26.17 & 24.33 & 3.36 & 3.24 \\
\hline 53 & NDSG-15 & 8.67 & 9.23 & 3.30 & 3.10 & 17.70 & 18.27 & 8.13 & 8.83 & 154.83 & 145.47 & 22.40 & 23.37 & 3.04 & 2.99 \\
\hline S. No & Genotypes & $\begin{array}{l}\text { Inter } \\
\text { nodal } \\
\text { length } \\
(\mathrm{cm})\end{array}$ & $\begin{array}{l}\text { Vine } \\
\text { length } \\
\text { (m) }\end{array}$ & $\begin{array}{l}\text { Fruit } \\
\text { length } \\
(\mathrm{cm})\end{array}$ & $\begin{array}{l}\text { Fruit } \\
\text { circumfer } \\
\text { ence }(\mathrm{cm})\end{array}$ & $\begin{array}{l}\text { Average } \\
\text { fruit } \\
\text { weight (g) }\end{array}$ & $\begin{array}{l}\text { No. of } \\
\text { fruits } \\
\text { per } \\
\text { plant }\end{array}$ & $\begin{array}{l}\text { Average } \\
\text { fruits } \\
\text { yield } \\
\text { per } \\
\text { plant } \\
\text { (kg) }\end{array}$ & S. No & $\begin{array}{l}\text { Genoty } \\
\text { pes }\end{array}$ & $\begin{array}{l}\text { Inter } \\
\text { nodal } \\
\text { length } \\
(\mathrm{cm})\end{array}$ & $\begin{array}{l}\text { Vine } \\
\text { length } \\
\text { (m) }\end{array}$ & $\begin{array}{l}\text { Fruit } \\
\text { length } \\
\text { (cm) }\end{array}$ & $\begin{array}{l}\text { Fruit } \\
\text { circumfer } \\
\text { ence }(\mathbf{c m})\end{array}$ & $\begin{array}{l}\text { Average } \\
\text { fruit } \\
\text { weight } \\
\text { (g) }\end{array}$ \\
\hline \multirow[t]{2}{*}{54} & Pusa Chikni @ & 4.17 & 5.03 & 2.90 & 2.73 & 27.57 & 25.47 & 8.80 & 9.13 & 156.55 & 164.87 & 24.63 & 22.43 & 3.24 & 3.18 \\
\hline & Mean & 6.86 & 6.88 & 3.34 & 3.32 & 23.75 & 23.95 & 8.76 & 8.76 & 145.75 & 145.05 & 24.66 & 24.35 & 3.04 & 3.04 \\
\hline \multirow{6}{*}{$\begin{array}{l}\text { Rang } \\
\text { e }\end{array}$} & Lowest & 3.57 & 4.10 & 2.07 & 1.93 & 15.87 & 17.37 & 7.87 & 7.50 & 95.53 & 100.60 & 9.97 & 10.33 & 1.45 & 1.48 \\
\hline & Highest & 9.00 & 9.23 & 6.37 & 6.43 & 32.50 & 33.00 & 9.83 & 10.60 & 200.83 & 190.10 & 37.63 & 38.60 & 3.95 & 4.35 \\
\hline & C.V. & 6.47 & 6.32 & 7.49 & 7.54 & 6.30 & 6.28 & 6.21 & 6.32 & 5.33 & 5.22 & 6.54 & 6.40 & 7.06 & 6.52 \\
\hline & S.E. & 0.26 & 0.25 & 0.14 & 0.14 & 0.86 & 0.87 & 0.31 & 0.32 & 4.49 & 4.37 & 0.93 & 0.90 & 0.12 & 0.11 \\
\hline & C.D. 5\% & 0.72 & 0.70 & 0.41 & 0.41 & 2.42 & 2.44 & 0.88 & 0.90 & 12.58 & 12.25 & 2.61 & 2.52 & 0.35 & 0.32 \\
\hline & C.D. 1\% & 0.95 & 0.93 & 0.54 & 0.54 & 3.20 & 3.22 & 1.17 & 1.19 & 16.65 & 16.21 & 3.45 & 3.34 & 0.46 & 0.42 \\
\hline
\end{tabular}

Table.3 Range of variation in mean values and grand means of various traits in sponge gourd $\left(\mathrm{Y}_{1}=2014\right.$ and $\left.\mathrm{Y}_{2}=2015\right)$

\begin{tabular}{|c|c|c|c|c|c|c|c|c|c|c|c|}
\hline \multirow{3}{*}{$\begin{array}{l}\text { S. } \\
\text { No. }\end{array}$} & \multirow[t]{3}{*}{ Characters } & \multicolumn{4}{|c|}{ Range of mean values } & \multicolumn{4}{|c|}{ Mean over } & \multirow{2}{*}{\multicolumn{2}{|c|}{ Grand mean }} \\
\hline & & \multicolumn{2}{|c|}{ Parents } & \multicolumn{2}{|c|}{ Crosses } & \multicolumn{2}{|c|}{ Parents } & \multicolumn{2}{|c|}{ Crosses } & & \\
\hline & & $\mathbf{Y}_{1}$ & $\mathbf{Y}_{2}$ & $\mathbf{Y}_{1}$ & $\mathbf{Y}_{2}$ & $\mathbf{Y}_{1}$ & $\mathbf{Y}_{2}$ & $\mathbf{Y}_{1}$ & $\mathbf{Y}_{2}$ & $\mathbf{Y}_{1}$ & $\mathbf{Y}_{2}$ \\
\hline 1 & $\begin{array}{l}\text { Node no.to anthesis of first staminate } \\
\text { flower }\end{array}$ & 3.00 to 13.40 & 2.67 to 14.13 & 3.03 to 6.03 & 3.07 to 5.77 & 6.66 & 6.78 & 4.47 & 4.48 & 5.03 & 5.08 \\
\hline 2. & $\begin{array}{l}\text { Node no.to anthesis of first pistillate } \\
\text { flower }\end{array}$ & 6.70 to 18.43 & 5.47 to 18.60 & 5.97 to 12.20 & 5.07 to 11.63 & 10.17 & 10.49 & 7.64 & 7.65 & 8.30 & 8.39 \\
\hline 3. & $\begin{array}{l}\text { Days to anthesis of first staminate } \\
\text { flower }\end{array}$ & 21.23 to 47.50 & 20.70 to 44.63 & 21.17 to 35.40 & 21.67 to 36.67 & 33.70 & 33.61 & 29.54 & 29.81 & 30.62 & 30.80 \\
\hline 4. & Days to anthesis of first pistillate flower & 26.63 to 43.10 & 29.10 to 46.53 & 25.47 to 42.17 & 23.87 to 46.53 & 36.80 & 36.98 & 33.56 & 34.55 & 34.40 & 34.55 \\
\hline 5. & Node no. of first fruit harvest & 7.20 to 18.77 & 8.00 to 18.77 & 6.13 to 13.30 & 6.30 to 12.40 & 10.97 & 11.15 & 8.35 & 8.23 & 9.03 & 8.99 \\
\hline 6. & Days to first fruit harvest & 40.80 to 54.57 & 41.47 to 56.50 & 38.17 to 55.23 & 37.50 to 54.63 & 48.26 & 48.74 & 45.42 & 45.68 & 46.15 & 46.47 \\
\hline 7. & No.of primary branches per plant & 4.07 to 9.07 & 3.73 to 9.17 & 4.20 to 8.43 & 4.27 to 8.60 & 6.27 & 6.68 & 6.31 & 6.42 & 6.30 & 6.49 \\
\hline 8. & Inter nodal length $(\mathrm{cm})$ & 3.57 to 9.00 & 4.10 to 9.23 & 4.13 to 8.97 & 4.37 to 9.17 & 6.27 & 6.17 & 7.06 & 7.12 & 6.86 & 6.88 \\
\hline 9. & Vine length $(\mathrm{m})$ & 2.07 to 6.17 & 1.93 to 5.93 & 2.17 to 6.37 & 2.17 to 6.43 & 3.35 & 3.27 & 3.34 & 3.34 & 3.34 & 3.32 \\
\hline 10. & Fruit length $(\mathrm{cm})$ & 15.87 to 31.23 & 17.37 to 31.00 & 17.67 to 32.50 & 18.23 to 33.00 & 23.74 & 23.13 & 23.75 & 24.24 & 23.75 & 23.95 \\
\hline 11. & Fruit circumference $(\mathrm{cm})$ & 7.87 to 9.83 & 7.80 to 10.60 & 7.90 to 9.83 & 7.50 to 10.07 & 8.65 & 8.75 & 8.80 & 8.77 & 8.76 & 8.76 \\
\hline 12. & Average fruit weight $(\mathrm{g})$ & 95.53to 190.23 & 100.60 to 174.53 & 99.70 to 200.83 & 105.40 to 190.10 & 142.12 & 140.37 & 147.02 & 146.69 & 145.75 & 145.05 \\
\hline 13. & No. of fruits per plant & 9.97 to 31.23 & 10.33 to 30.33 & 16.17 to 37.63 & 15.27 to 38.60 & 22.58 & 21.85 & 25.39 & 25.22 & 24.66 & 24.35 \\
\hline 14. & Average fruits yield per plant $(\mathrm{kg})$ & 1.45 to 3.39 & 1.48 to 3.51 & 2.17 to 3.95 & 2.08 to 4.35 & 2.68 & 2.67 & 3.17 & 3.17 & 3.04 & 3.04 \\
\hline
\end{tabular}


Partitioning of variances into lines revealed highly significant differences for all the traits and variances due to testers were also highly significant for all the traits in both the years (Table 1). The highest average mean performance for average fruit yield per plant along with some of the component traits was exhibited by parental genotypes NDSG-21 (3.39 and $3.51 \mathrm{~kg}$ ) followed by NDSG-12 (3.36 and $3.24 \mathrm{~kg}$ ), NDSG-1 (3.28 and 3.13 $\mathrm{kg}$ ), Pusa Chikni (3.24 and $3.18 \mathrm{~kg}$ ) and NDSG-15 (3.04 and $2.99 \mathrm{~kg}$ ) and Some other parental genotypes exhibiting very high mean performance for other characters than average fruit yield per plant (Table 2) were NDSG-18 (40.80 and 41.47 days), NDSG-10 (41.47 and 42.73 days) and NDSG-11 (43.60 and 42.50 days) in both the years for days to first fruit harvest; NDSG-18 (31.23 and 29.20), NDSG55 (29.57 and 26.07) and NDSG-1 (26.53 and 30.33 ) for number of fruits per plant, NDSG-6 (31.23 and 29.30), NDSG-2 (30.07 and 31.00) and Pusa Chikni (27.57 and 25.47) in both the years for fruit length and NDSG-10 (190.23 and 174.53), NDSG-4 (163.57 and 153.60) and Pusa Chikni (156.55 and 164.87) in both the years for average fruit weight.

Same result observed by Sabina et al., (2008), Sanandia et al., (2014), Tamil selvi et al., (2015). Some hybrids given desirable mean performance for average fruit yield per plant along with some of the component traits was exhibited by NDSG-55 × NDSG-11 (3.95 and $4.23 \mathrm{~kg}$ ) followed by NDSG-63 $\times$ Pusa Chikni (3.95 and $4.35 \mathrm{~kg}$ ), NDSG-24 $\times$ Pusa Chikni (3.89 and $4.12 \mathrm{~kg}$ ), NDSG-18 $\times$ Pusa Chikni (3.79 and $4.06 \mathrm{~kg}$ ) and NDSG-18 $\times$ NDSG-11 (3.87 and $4.01 \mathrm{~kg})$ in both the years.

The hybrid NDSG-18 $\times$ NDSG-11 took minimum number of days to first fruit harvest (38.17 days) followed by NDSG-10 × NDSG11 (38.23) and NDSG-55 × NDSG-11 (37.50 days) followed by NDSG-4 $\times$ NDSG-11
(38.50) in $Y_{1}$ and $Y_{2}$, respectively. The significantly maximum number of fruits per plant was observed in NDSG-18 $\times$ NDSG-11 (37.63 fruits) which was followed by NDSG$18 \times$ Pusa Chikni (34.43) and NDSG-18 $\times$ NDSG-11 (38.60 fruits) which was followed by NDSG-18 $\times$ Pusa Chikni (37.50) in $\mathrm{Y}_{1}$ and $\mathrm{Y}_{2}$, respectively. While, the havier fruit weight was exhibited by the NDSG-10 $\times$ NDSG-15 $(200.83 \mathrm{~g})$ and NDSG-4 $\times$ Pusa Chikni $(186.40 \mathrm{~g})$ and NDSG-6 $\times$ Pusa Chikni $(190.10 \mathrm{~g})$ and NDSG-10 $\times$ NDSG-15 (187.47 g) $\mathrm{Y}_{1}$ and $\mathrm{Y}_{2}$, respectively.

Range of variation in mean values and grand means of various traits are given in table 3 . These genotypes (parents and hybrids) merits due consideration as promising parents for hybridization programme for bringing over all improvement in plant architecture in a component breeding approach ultimately leading to high yielding sponge gourd genotypes even if they have moderate or low fruit yield.

\section{Acknowledgement}

The authors are thankful to the HOD, Department of Vegetable Science, NDUA\&T, Kumarganj, Faizabad (U.P.) for providing seed material and facilities for conducting research.

\section{References}

Kalloo, G. (1993). Loofah-Luff S.P.P. (Ed.). Genetic Improvement of Vegetable Crops. pp. 265-266.

Panse, V.G. and Shukhatme, P.V. (1967). Statistical Methods for Agricultural Workers, $2^{\text {nd }}$ edn. Indian Council of Agriculture Research, New Delhi, pp. 235-247.

Rai, M. and Pandey, A.K. (2007). Towards a rainbow revolution. The Hindu Survey of Indian Agril. Pp.112-119. 
Sabina Islam Munshi, A. D.; Ravinder Kumar Behera, T. K. and Lal, S. K. (2008). Evaluation of sponge gourd hybrids for yield and related traits. Cucurbit Genetics Cooperative, (31/32): 34-35.

Sanandia, S.T.; Mehta, D.R. and Tarpara V.D. (2014). Inheritance of fruit yield and its components in sponge gourd [Luffa cylindrica (Roem) L.]. Department of Horticulture, Junagadh Agricultural University, Junagadh-362 001 (India). Veg. Sci. 37(1): 25-29.

Singh, K. (2004). Transformation of Vegetable Science in India-Looking Back and Ahead. Financing Agriculture. Oct. to Dec., pp. 15-28.
Tamil selvi1, N.A.; Jansirani, P. and Pugalendhi L. (2015). Line $x$ Tester analysis for yield and its component traits in pumpkin (Cucurbita moschata Duch. Ex Poir). Department of Vegetable Crops, Tamil Nadu Agricultural University, Coimbatore641 003. Electronic Journal of Plant Breeding, 6(4): 1004 -1010.

Whitaker, T.W. and Davis, G.N. (1962). Cucurbits. Interscience Publ. Inc. New Yark. Pp. 250.

Yawalkar, K.S. (2004). Cucurbitaceous or vine crops. Vegetable crops of India (V.ed.) p.152-155.

\section{How to cite this article:}

Yamuna Prasad Singh, V.B. Singh, Praveen Kumar Singh, Vimlesh Kumar, Maneesh Pandey and Gyanendra Singh. 2017. Studies on Mean Performance for Yield and its Contributing Traits of Sponge Gourd [Luffa cylindrica (Roem) L.]. Int.J.Curr.Microbiol.App.Sci. 6(8): 11701179. doi: https://doi.org/10.20546/ijcmas.2017.608.145 